\title{
EVALUACIÓN DE CALIDAD DE UN SERVICIO DE VOLUNTARIADO HOSPITALARIO EN ONCOLOGÍA: NIVELES DE SATISFACCIÓN DE LOS PACIENTES DE CÁNCER Y SUS FAMILIAS
}

\author{
EVALUATION OF QUALITY OF VOLUNTEER SERVICE IN ONCOLOGY: LEVELS OF \\ SATISFACTION OF CANCER PATIENTS AND THEIR FAMILIES
}

\author{
Belén Fernández Sánchez y Adoración Martínez \\ Asociación Española Contra el Cáncer
}

Resumen

Objetivo y método: evaluar el impacto del programa de voluntariado hospitalario a través de la importancia atribuida, el grado de satisfacción manifestado por los usuarios y la disposición a recomendar el servicio. Para ello se realizó un estudio de satisfacción realizado en dos fases. Estudio cuantitativo a través de entrevista personal con cuestionario semiestruturado y estudio cualitativo a través de entrevista personal abierta.

Resultados: Participaron 654 pacientes y familiares. En la valoración de la actividad voluntaria se obtiene una puntuación de $(9,3)$ en pacientes $(9,2)$ en familiares en una escala de 0 a 10. El $77 \%$ de los usuarios se muestra dispuesto a recomendar a otras personas el servicio de voluntariado obteniéndose un índice NPS netamente positivo $(+80)$. Los usuarios destacan de los voluntarios especialmente la amabilidad y el respeto por la intimidad $(89 \%$ y el $82 \%$ respectivamente) y ofrecen una puntuación media de 9,2, y 9,4 en una escala de 10.

Conclusiones: Se obtiene una valoración unánimemente positiva de la labor de los voluntarios y una gran disposición a recomendar el contacto con los voluntarios. La mayor utilización de los servicios de los voluntarios se vincula a una mejor valoración. Se concede mayor prioridad a la actividad de visitar a pa-
Abstract

Objective and method: to evaluate the impact of the program for voluntary work at hospitals through the importance assigned, satisfaction level stated by users, and willingness to recommend the service. For this purpose, a satisfaction study was carried out in two phases: A quantitative study through personal interviews with semi-structured questionnaire and a qualitative study through open personal interviews.

Results: 654 patients and family members took part. In the evaluation of volunteers, a rating of 9.3 is given by patients and 9.2 by family members, in a scale from 0 to $\mathbf{1 0}$. Seventy-seven percent of users is willing to recommend the volunteer service to others, achieving a clearly positive NPS index $(+80)$. Users specially emphasize from volunteers their kindness and their respect for privacy $(89 \%$ and $82 \%$ respectively) and provide an average rating of 9.2 and 9.4 in a scale of 0 to 10 .

Conclusions: Unanimous positive evaluation of the work by volunteers and great willingness to recommend the contact with volunteers is obtained. The increased use of the volunteer service is linked to better results achieved in the evaluation. Greater priority is given to the activity of visiting patients in hospitals to keep company and provide emotional support.

Correspondencia:

Belén Fernández Sánchez,

Asociación Española Contra el Cáncer

C/Amador de los Ríos, 5. 28010 Madrid

E-mail: belen.fernandez@aecc.es 
cientes ingresados para acompañar y ofrecer apoyo emocional. La valoración de la labor desempeñada por los voluntarios es muy positiva especialmente en amabilidad y respeto por la intimidad. Los usuarios no demandan nada nuevo pero si incrementar la difusión del servicio, más voluntarios y ampliar el horario de atención.

Palabras clave: cáncer, evaluación, voluntariado, hospital, satisfacción.
The evaluation of the work performed by the volunteers is very positive, especially regarding kindness and respect for privacy. Rather than new specific requirements, users demand an increased dissemination of the service, more volunteers and extended service hours.

Keywords: Cancer, evaluation, volunteer, hospital, satisfaction

\section{INTRODUCCIÓN}

La acción voluntaria en oncología se manifiesta como una necesidad fundamental ya que en la problemática del cáncer se encuentran implicados múltiples aspectos físicos, psicológicos y sociales que no pueden ser satisfechos única y exclusivamente con una buena infraestructura de servicios sanitarios. Para ofrecer una respuesta completa a los enfermos y familiares es necesario contemplar otros elementos como el afecto, la compresión, la distracción, la compañía; aspectos a los que las administraciones no pueden llegar y sí pueden ser satisfechos por la intervención de los voluntarios.

En numerosos estudios se señala que en condiciones de estrés elevado, el apoyo social protege a los individuos de los efectos negativos sobre el estado de ánimo y el funcionamiento psicológico, facilitando los procesos de afrontamiento y adaptación. Asimismo, la literatura refiere que los pacientes con un diagnóstico de cáncer presentan un menor malestar emocional y una mayor adaptación al proceso de enfermedad y tratamiento si poseen vínculos sociales o familiares que les permitan afrontar de manera menos amenazante su enfermedad ${ }^{(1-4)}$. Algunos estudios realizados sobre la influencia del apoyo social en el ajuste a la enfermedad muestran que las intervenciones basadas en el apoyo social tienen efectos positivos en el bienestar de los pacientes porque movilizan los recursos informales promoviendo la participación, aumentan la capacidad de afrontar situaciones de estrés e incrementan las competencias personales ${ }^{(1,5)}$. El apoyo social es un elemento de gran relevancia para el bienestar de los individuos a la hora de hacer frente a las enfermedades oncológicas $^{(1,6-9)}$. De Leeuw et al. ${ }^{(10)}$ examinan la influencia del apoyo social en la sintomatología depresiva de pacientes con cáncer de cabeza y cuello que tienen que enfrentarse no sólo a un diagnóstico amenazante para su vida, sino también a una apariencia física desfigurada y a la pérdida o deterioro de funciones importantes. Los datos muestran que el apoyo recibido está asociado con más sintomatología depresiva de base, mientras que el apoyo disponible implica menor severidad de los síntomas depresivos. Levy et al., realizaron un estudio con mujeres diagnosticadas de cáncer de mama y encontraron que el apoyo social percibido predecía el afrontamiento adecuado a la enfermedad. Otros trabajos realizados con mujeres diagnosticadas de cáncer de mama han revelado que el apoyo social mitiga el malestar emocional derivado de la enfermedad ${ }^{(11,12)}$. Devine et al. examinan la asociación entre el apoyo social y el nivel de pensamientos intrusivos y de evitación en pacientes con melanoma metastático y cáncer metastático de 
células renales. Los datos evidencian que el apoyo social se asocia negativamente con el malestar emocional y positivamente con la calidad de vida ${ }^{(13)}$. Sin duda, los datos apoyan la necesidad de desarrollar intervenciones dirigidas a incrementar el nivel de apoyo interpersonal de pacientes y familiares, a través de diferentes redes de voluntariado, para facilitar la adaptación de los mismos a la enfermedad.

Consciente de los beneficios derivados del apoyo social para enfermos de cáncer y sus familiares, la Asociación Española Contra el Cáncer (AECC) desde sus inicios ha puesto en marcha servicios de apoyo a enfermos. Destacar, que el voluntariado constituye una parte muy importante dentro de la entidad, tanto por su participación en actividades de órgano de gobierno como en todas aquellas realizadas directamente en beneficio del enfermo de cáncer y sus familiares en programas de atención hospitalaria, domiciliaria, residencias y pisos de acogida, unidades de cuidados paliativos, etc. La AECC tiene un total de 20.298 voluntarios, un $28 \%$ de los mismos, concretamente 5.731, desarrollan actividades de apoyo y acompañamiento de enfermos y familiares. Prácticamente la totalidad de dichas actividades se desarrolla en el ámbito hospitalario, con im- plantación en 170 hospitales en el ámbito nacional. En la mayoría de los hospitales los voluntarios se encuentran en el servicio de oncología y en el hospital de día (79\%). Respecto a las tareas desarrolladas por los voluntarios en los diferentes hospitales, tal como aparece en la tabla 1, destacar que en casi todos ellos, en el 92\% de los casos, realizan visitas a los ingresados en planta.

Los servicios de atención y acompañamiento desarrollados por el voluntariado en el ámbito oncológico cada día son más importantes y numerosos ${ }^{(14)}$. En el momento actual, a medida que se van mejorando las tasas de implantación de los voluntarios en el ámbito hospitalario, el reto ineludible para las entidades de voluntariado es la apuesta por la calidad. Como prestadores de servicios psicosociales, estas entidades deben dedicar especial atención a establecer indicadores de calidad asistencial y evaluar de forma sistemática y objetiva los resultados derivados de su desempeño. Es necesario establecer un sistema de indicadores, integrado en un modelo de aprendizaje organizativo, que a través de la definición de objetivos e iniciativas estratégicas supongan un feedback de mejora continua. Es básico que el sistema de calidad que se utilice ofrezca información sobre las preferencias, las expectativas, so-

\section{Tabla 1. Actividades desarrolladas por los voluntarios de la AECC en los hospitales}

\begin{tabular}{lcc}
\hline Actividades desarrollada por los voluntarios & $\mathbf{N}^{\mathbf{0}}$ hospitales & \\
\hline Acogida con el carrito de desayunos & 65 & $56 \%$ \\
Visitas en planta & 108 & $92 \%$ \\
Relevo del cuidador principal & 3 & $3 \%$ \\
Guía hospitalaria & 86 & $73 \%$ \\
Gestiones y trámites hospitalarios & 79 & $67 \%$ \\
Actividades de animación & 48 & $41 \%$ \\
Talleres educativos & 30 & $26 \%$ \\
Sesiones informativas sobre los servicios de la aecc & 58 & $50 \%$ \\
\hline
\end{tabular}


bre qué aspectos son más relevantes en cada momento, sobre cómo modificar la organización hacia las necesidades cambiantes de los usuarios ${ }^{(15,16)}$.

En este sentido, la satisfacción de los beneficiarios es un objetivo irrenunciable de cualquier institución ${ }^{(17)}$. Sus opiniones, así como la valoración de sus niveles de satisfacción, se constituyen en un poderoso instrumento para la mejora de la calidad asistencial y para adecuar el servicio a las necesidades reales de los usuarios ${ }^{(18)}$. El papel de la satisfacción como mecanismo de suministro de información relevante para la toma de decisiones es incuestionable en las organizaciones de salud, que destacan por la diversidad de servicios, actividades y ámbitos de desarrollo. En cuanto a las razones por las que medir la satisfacción, los diferentes autores las han resumido en cuatro grandes categorías: (1) se constituye en un feedback imprescindible para la gestión y optimización de los recursos; (2) ofrece una medida fiable de la capacidad de la organización para satisfacer las necesidades de los usuarios, pudiendo ser utilizado como un indicador en el Control de Calidad del servicio; (3) es uno de los aspectos con los que cuentan las organizaciones para lograr mejoras y resultados estables a medio plazo; y, por último, (4) es un predictor de las reacciones o comportamiento de los usuarios (lealtad, prescripción, adherencia al tratamiento, etc. $)^{(19)}$.

En la AECC hay una larga tradición de evaluación, en este sentido anualmente se aplica un protocolo para valorar la satisfacción del paciente y el familiar con la atención recibida a través del voluntariado, así como la satisfacción de los profesionales sanitarios con la actividad del voluntariado en el hospital. Dicha evaluación se ha venido realizado mediante encuestas de satisfacción y nos ha permitido analizar las percepciones de paciente, familiares y profesionales con respecto a determinados aspectos del servicio ofreci- do por los voluntarios. Si bien, la identificación de los puntos fuertes y débiles del funcionamiento del servicio es un elemento clave para la mejora de los procesos, resulta incompleto en la medida que no establece un mapa de prioridades de los diferentes aspectos que lo configuran. En este contexto y con el objetivo de superar dicho hándicap, durante el 2015, se ha realizado una evaluación en las que se han combinado las percepciones de los usuarios con la importancia que otorgan a los diferentes aspectos del servicio que han recibido. Este enfoque metodológico, muy utilizado en el ámbito de la valoración de la satisfacción de los usuarios de los servicios de salud, nos ha permitido identificar las áreas de excelencia y de mejora así como definir actuaciones para optimizar el desempeño del servicio de voluntariado.

El presente estudio se enmarca dentro de esta aproximación metodológica y tiene como objetivos: 1) evaluar el impacto del programa de voluntariado hospitalario. Determinar hasta qué punto las acciones desarrolladas influye positiva o negativamente en la calidad de la ayuda y en la tasa de satisfacción de los usuarios e identificar áreas de mejora. 2) optimizar el proceso de seguimiento de los programas de voluntariado, minimizando el efecto de las variables que influyan negativamente en el grado de satisfacción de los diferentes colectivos implicados, pacientes diagnosticados de cáncer y sus familiares

\section{MÉTODO}

\section{Muestra}

La muestra utilizada en el presente estudio estuvo compuesta por un total de 654 usuarios de 14 hospitales de la Red Nacional de Salud (404 pacientes y 250 familiares). Se incluyeron pacientes de cáncer mayores de 18 años en cualquier fase de la enfermedad que hubiesen tenido contacto 
con los voluntarios de la AECC (quedando excluidos los pacientes cuya calidad de vida actual dificultará su participación en el estudio) así como familiares de pacientes que hubiesen recibido algún tipo de atención por parte de los voluntarios. El criterio de selección de los participantes ha sido, a partir del cumplimiento de las condiciones del target, el azar, realizándose determinadas preguntas filtro para concretar el cumplimiento de los requisitos.

En cuanto a las características generales de la muestra, el $62 \%$ de los usuarios era paciente y el $38 \%$ restante familiar. El número de mujeres ha sido muy superior al de varones (un $65 \%$ y un $35 \%$, respectivamente). Los participantes tenían una media de edad de 50 años ( $S D=13,3$ años, rango 18-90 años). La distribución de los pacientes y familiares en los diferentes intervalos de edad fueron los siguientes; entre 18 y 30 años (3\%), entre 31 y $40(19 \%)$, entre 41 y $50(26 \%)$, entre 61 y $70(26 \%)$ y más de 70 años (15\%). Lo que significa que un $52 \%$ de beneficiarios se encontraban en el intervalo de edad de 51 a 70 años.

\section{Procedimiento}

Mediante una entrevista personal en la sala de espera y en las plantas de oncología de los centros hospitalarios se cumplimentó un cuestionario estructurado para pacientes y familiares. Tras concluir el estudio cuantitativo se realizó un estudio cualitativo, con el objeto de identificar áreas de mejora a través de 4 entrevistas personales realizadas con un cuestionario abierto.

Para la elaboración de la escala, fue preciso en primer lugar, definir las dimensiones relevantes al servicio de atención y acompañamiento realizado por los voluntarios. Para ello se pusieron en marcha dos estrategias complementarias:

1. Revisión de la bibliografía sobre evaluación de calidad de servicios de salud. Como resultado de esta búsqueda se identificaron un grupo de criterios utilizados en evaluación de satisfacción en servicios sanitarios (tanto en el ámbito nacional como en el internacional), aunque no se ha encontrado ningún modelo conceptual prexistente que se pudieran importar de manera directa a la evaluación de la atención ofrecida por los voluntarios. Apenas hay experiencias en el desarrollo de herramientas de evaluación para decidir qué servicios de voluntariado deberán ser prioritarios en la práctica diaria. Este es nuestro caso, y por lo tanto fue necesario desarrollar un sistema ad hoc de valoración, con la finalidad última de desarrollar posteriormente una escala que dote de una mayor calidad y equidad a las prestaciones realizadas por los voluntarios.

2. Definición de los criterios a través de un grupo de discusión constituido por profesionales que se dedican a la coordinación de voluntariado en la AECC y profesionales expertos en evaluación de investigación de mercados y consultoría en marketing estratégico de la empresa IKERFEL. El grupo estuvo constituido por psicólogos, trabajadores sociales y responsables de administración y gestión.

La escala resultante está integrada por tres bloques temáticos con una variedad de contenidos e ítems cada uno de ellos:

- Información y utilidad de las acciones de voluntariado. A través de los ítems que integran este bloque temático podemos conocer el nivel de información que tienen tanto pacientes como familiares sobre las actividades que desarrollan los voluntarios en el hospital y su opinión sobre la importancia y utilidad de cada una de ellas. Por último, en este capítulo, se evalúa la recomendación de los servicios desarrollados por los voluntarios. 
- Calidad de las acciones de voluntariado. Nos permite conocer la calidad percibida por parte de pacientes y familiares sobre el servicio de voluntariado. En este bloque se incluyen las siguientes variables: Fiabilidad (habilidad para ejecutar el servicio de forma fiable y cuidadosa); Capacidad de respuesta (disposición y voluntad para ayudar al beneficiario); Seguridad (conocimiento mostrado por los voluntarios y su habilidad para inspirar credibilidad y confianza); Empatía (atención Individualizada y capacidad de compresión y aceptación que ofrecen los voluntarios a los usuarios)

- Áreas de mejora. Recoger propuestas para mejorar las actuaciones de los voluntarios.

\section{RESULTADOS}

En la mayoría de los hospitales los voluntarios se encuentran en el servicio de oncología y en el hospital de día, en una proporción del $79 \%$ en ambos casos. Seguidamente se señalan los servicios de hematología (56\%) y consultas externas (44\%). Respecto a las actividades desarrolladas por los voluntarios en los diferentes hospitales, destacar que en casi todos ellos, en el $92 \%$ de los casos, realizan visitas a los ingresados en planta. Por el contrario, el tipo de actividad menos desarrollada es el relevo del cuidador principal sólo señalada en el 3\% de los casos.

Los servicios más conocidos y utilizados son la visita en planta a pacientes para facilitar apoyo emocional, la acogida a pacientes y familiares a través del carro de desayuno y la información sobre servicios de la AECC Así, el 84\% de los pacientes y familiares presenta un elevado conocimiento de la actividad "Visitar a los pacientes ingresados" y el 53\% un nivel medio de conocimiento de la actividad "carrito de acogida".

Dos de cada diez entrevistados cita espontáneamente la actividad de informar sobre los servicios que la aecc ofrece a los pacientes" y el resto de tareas muchos más instrumentales y prácticas son citadas por menos de 2 entrevistados de cada 10: "talleres educativos", "Entretener a pacientes y familiares", "Acompañar en desplazamientos por el hospital", "Suplir temporalmente a los familiares en el acompañamiento del enfermo para proporcionarles descanso". Tareas que por otra parte han sido valoradas de forma muy positiva por los beneficiarios. Una mayor utilización de los servicios de los voluntarios se vincula a una relativa mejor valoración.

Hay una valoración unánimemente positiva de la labor de los voluntarios. No se observan diferencias en la valoración que hacen los pacientes $(9,3)$ y sus familiares $(9,2)$. A mayor edad de los familiares esa valoración es más positiva (media de 8,8 en familiares de menos de 40 años a 9,5 entre los que tienen más de 70). Los datos señalan una valoración claramente positiva de todas y cada una de las tareas desarrolladas por los voluntarios (tabla 2), dado que en todas ellas se ha alcanzado al menos una puntuación de 8 en escala 0 a 10).

La valoración positiva también se manifiesta en la gran disposición a recomendar el contacto con los voluntarios. Así, un $77 \%$ se ha mostrado dispuesto a recomendar a otras personas el servicio de voluntariado. Para valorar el nivel de recomendación se ha utilizado el índice NPS (Net Promoter Score), introducido por F. Reichheld en $2003^{(20)}$, es un indicador que mide la satisfacción con respecto a un producto o servicio. En una escala de 0 (muy improbable) a 10 (sin duda lo recomendaría), se suman las respuestas de acuerdo a estos rangos: De 9 a 10 = "Promotores"; De 7 a 8 = "Pasivos"; 
De 0 a $6=$ "Detractores". Si restamos el porcentaje de "detractores" al porcentaje de "promotores", se obtiene un resultado que puede ser tan bajo como -100 (todos han calificado 6 o menos) o tan alto como 100 (todos han calificado 9 o 10). Un NPS superior a 0 se percibe como bueno y un NPS de 50 o más es excelente. En nuestro caso, tal como se muestra en la tabla 3, se ha obtenido un Índice NPS netamente positivo (+80). Además no presenta diferencias significativas en los diferentes segmentos poblacionales considerados.

\section{Tabla 2. Valoración de las actividades desarrolladas por los voluntarios de la AECC en los hospitales}

\begin{tabular}{|c|c|c|c|c|c|c|c|c|}
\hline & $\begin{array}{c}\text { Visitar a } \\
\text { pacientes }\end{array}$ & $\begin{array}{c}\text { Actividades } \\
\text { lúdicas }\end{array}$ & $\begin{array}{c}\text { Traslados } \\
\text { hospital }\end{array}$ & $\begin{array}{c}\text { Realizar } \\
\text { gestiones }\end{array}$ & $\begin{array}{c}\text { Informar } \\
\text { servicios }\end{array}$ & $\begin{array}{c}\text { Talleres } \\
\text { educativos }\end{array}$ & $\begin{array}{c}\text { Suplir a } \\
\text { familiares }\end{array}$ & $\begin{array}{c}\text { Carrito } \\
\text { acogida }\end{array}$ \\
\hline Media & 9,1 & 8,1 & 8,6 & 8,6 & 8,6 & 8,3 & 8,9 & 8,6 \\
\hline
\end{tabular}

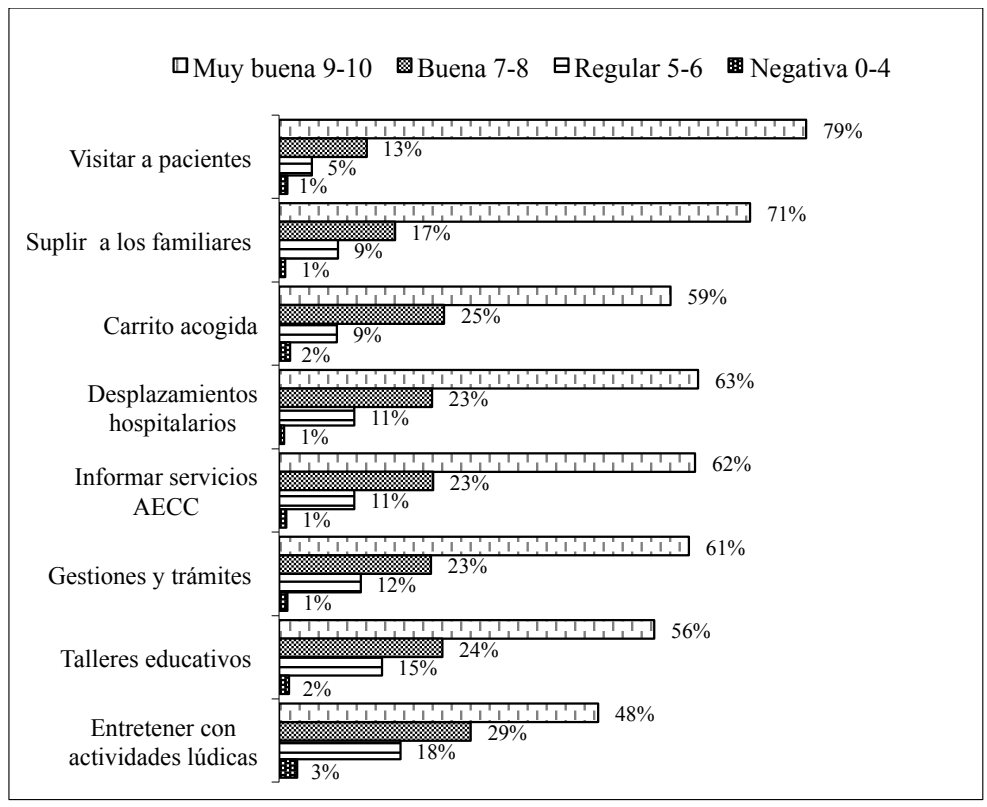

\section{Tabla 3. Índice NPS de las actividades desarrolladas por los voluntarios de la AECC en los hospitales}

\begin{tabular}{|l|c|c|c|}
\hline & PACIENTES & FAMILIARES & TOTAL \\
\hline Promotores (valoraciones 9 y 10) & $84 \%$ & $83 \%$ & $83 \%$ \\
\hline Pasivos (valoraciones 7 y 8) & $13 \%$ & $13 \%$ & $13 \%$ \\
\hline Detractores (valoraciones de 0 a 6) & $2 \%$ & $3 \%$ & $3 \%$ \\
\hline ÍNDICE NPS (promotores - detractores) & $\mathbf{+ 8 2}$ & $\mathbf{+ 8 0}$ & $\mathbf{+ 8 0}$ \\
\hline
\end{tabular}


Respecto a la importancia relativa de cada una de las acciones desarrollada por los voluntarios, en la figura 1 se muestra la importancia atribuida tanto por pacientes y familiares a cada una de ellas.

Se define con claridad como la tarea más importante la de "visitar a los pacientes para acompañarles y ofrecerles apoyo emocional". Ello es así tanto en el universo de los pacientes como también en el de los familiares (el $66 \%$ y el $58 \%$ respectivamente). El segundo aspecto más señalado ha sido "suplir temporalmente a los familiares en el acompañamiento del enfermo". Dicha tarea se ha citado en mayor medida como la más importante entre los familiares (el $7 \%$ de los pacientes frente al $14 \%$ de familiares. Idénticos resultados se constatan cuando se obtiene la importancia ponderada de las diferentes tareas, (tabla 4).

Tal como se muestra en la figura 2 se ha establecido un mapa de posicionamiento de las diferentes tareas atendiendo a la valoración que los usuarios realizan de

\section{Figura 1. Importancia atribuida tanto por pacientes y familiares a las diferentes actividades desarrolladas por los voluntarios}

\begin{tabular}{|c|c|}
\hline & \\
Visitar a pacientes ingresados para saludar, & \\
acompañar y ofrecerles apoyo emocional & \\
Suplir temporalmente a los familiares en en \\
acompañamiento del enfermo para \\
proporcionarles descanso
\end{tabular}

Tabla 4. Importancia ponderada de las actividades desarrolladas

\begin{tabular}{|c|c|c|c|c|c|c|c|}
\hline $\begin{array}{c}\text { Visitar a } \\
\text { pacientes }\end{array}$ & $\begin{array}{c}\text { Actividades } \\
\text { lúdicas }\end{array}$ & $\begin{array}{c}\text { Traslados } \\
\text { hospital }\end{array}$ & $\begin{array}{c}\text { Realizar } \\
\text { gestiones }\end{array}$ & $\begin{array}{c}\text { Informar } \\
\text { servicios } \\
\text { AECC }\end{array}$ & $\begin{array}{c}\text { Talleres } \\
\text { educativos }\end{array}$ & $\begin{array}{c}\text { Suplir a } \\
\text { familiares }\end{array}$ & $\begin{array}{c}\text { Carrito } \\
\text { acogida }\end{array}$ \\
\hline $37 \%$ & $5 \%$ & $11 \%$ & $11 \%$ & $9 \%$ & $6 \%$ & $16 \%$ & $5 \%$ \\
\hline
\end{tabular}


las mismas y al porcentaje de importancia atribuido a cada una de ellas. Las tareas consideradas como especialmente importantes y mejor valoradas son "Visitar a los enfermos para ofrecerles apoyo emocional" y "Suplir temporalmente a los familiares".

Respecto a la calidad de las acciones de voluntariado, la valoración de la labor desempeñada por los voluntarios es muy positiva en todas las variables consideradas. En este contexto, en la figura 3 se muestra que los beneficiarios destacan especialmente la amabilidad, así el $89 \%$ de los pacientes y familiares están totalmente de acuerdo con esta afirmación y ofrecen una puntuación media de 9,2, en una escala de 0 a 10. El respeto por la intimidad y confidencialidad ha recibido el $82 \%$ del acuerdo, obteniendo una puntuación media de 9,4. Las va- loraciones positivas aunque inferiores (respecto del promedio general) se han dado a los horarios y al cumplimiento de las tareas en un tiempo determinado. No hay diferencia entre la valoración que hacen pacientes y los familiares.

Finalmente, respecto a las áreas de mejora del servicio de voluntariado hospitalario un $87 \%$ no cita ningún aspecto a mejorar. Sólo un $10 \%$ de los usuarios manifiestan que les gustaría que los voluntarios desarrollen otras actividades que actualmente no realizan. No hay diferencias al respecto entre lo que es la opinión de los pacientes y la de sus familiares. Como áreas de mejora señalan una mayor difusión de la actividad, el incremento del número de voluntarios y la ampliación del horario de atención.

\section{Figura 2. Mapa de posicionamiento atendiendo a la importancia y valoración de las diferentes tareas desarrolladas por los voluntarios}

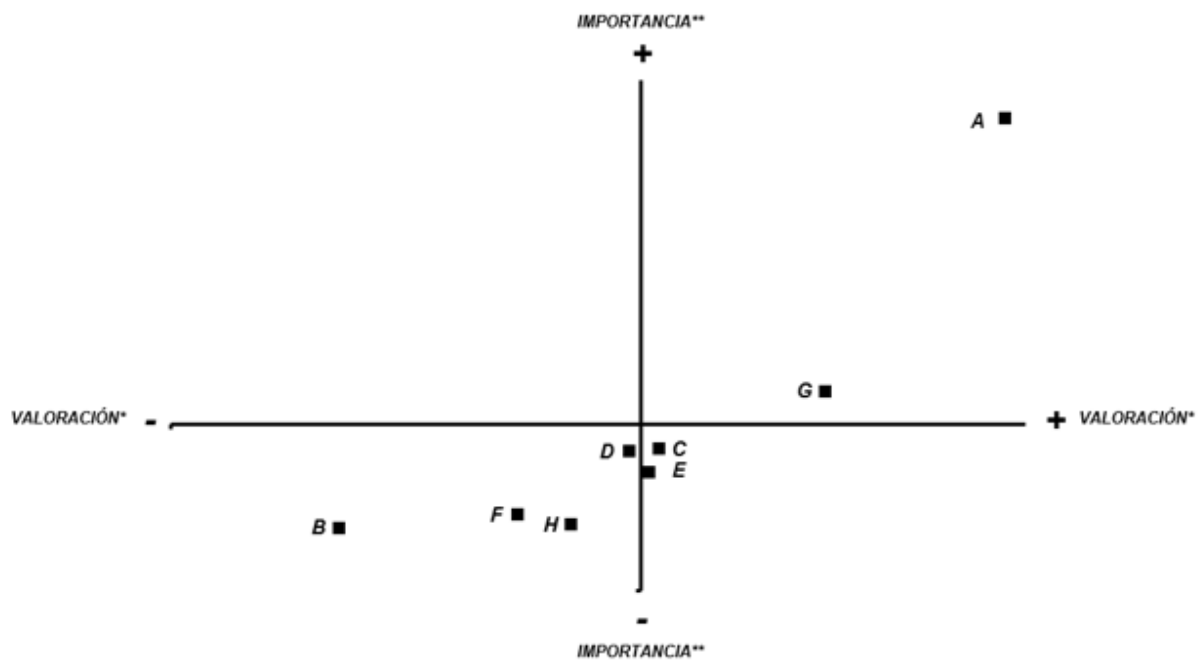

A. Visitar a pacientes ingresados para saludar, acompañar y ofrecerles apoyo emocional

B. Entretener a los pacientes y familiares con actividades lúdicas, manualidades

C. Acompañar a enfermos y familiares en sus desplazamientos dentro del hospital

D. Realizar gestiones y trámites hospitalarios para los pacientes (citaciones, etc...)

E. Informar sobre los servicios que le AECC ofrece a los pacientes y familiares

F. Desarrollar talleres educativos (pautas de alimentación, cuidados estéticos, etc....)

G. Suplir temporalmente a los familiares en el acompañamiento del enfermo

H. Carrito de acogida 
Figura 3. Valoración de la calidad de las acciones de voluntariado

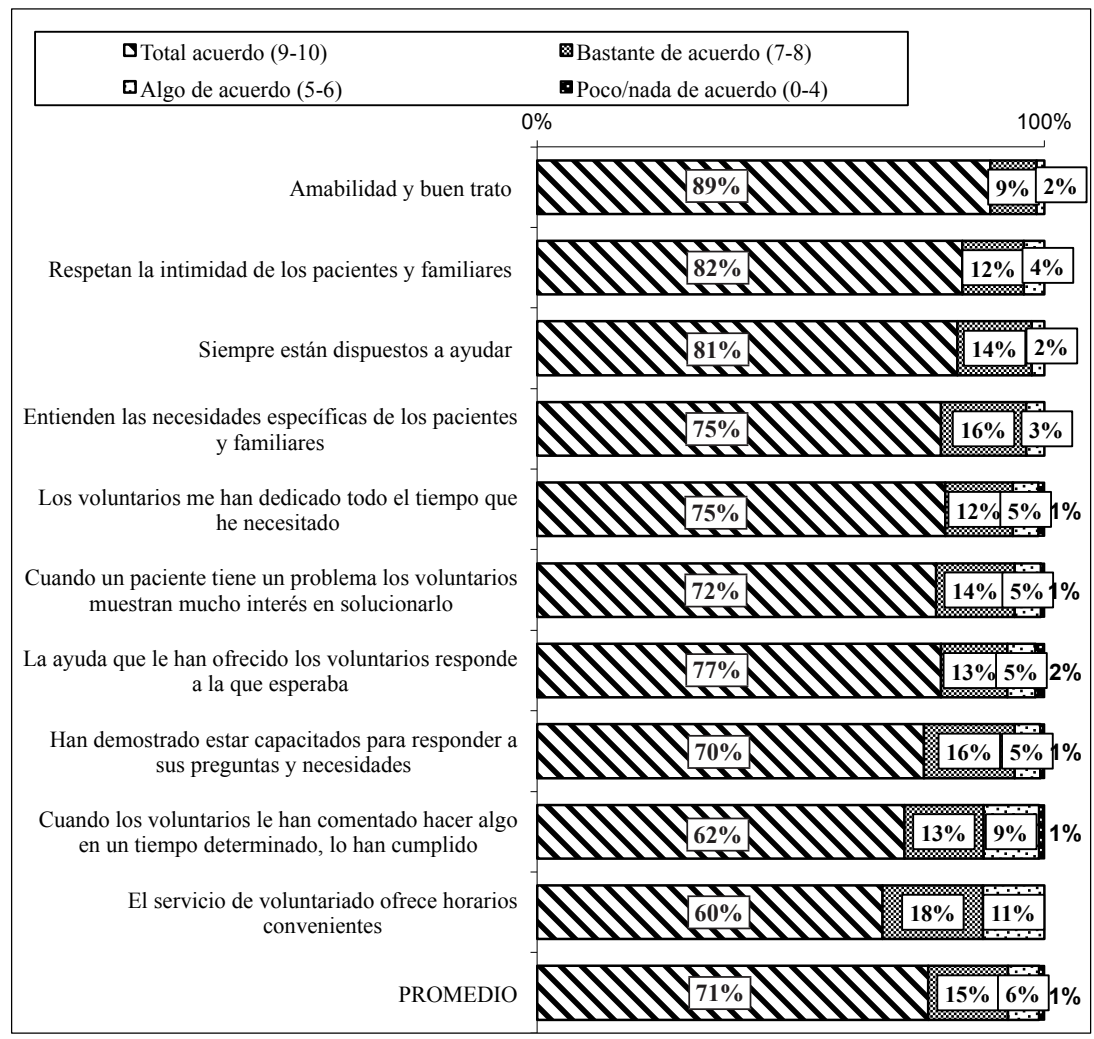

\section{DISCUSIÓN}

Aunque no son muy numerosos los estudios que ahondan en qué aspectos de la asistencia desarrollada por los voluntarios son los más y menos valorados por los pacientes, existe un cierto nivel de consenso sobre aquellos aspectos que se consideran más importantes. En este sentido podemos destacar: la accesibilidad, la competencia de los voluntarios a la hora de desarroIlar la atención, el resultado de la atención prestada, la empatía, la capacidad de respuesta. No obstante, parece aconsejable identificar claramente aquellas dimensiones de evaluación que resulten particularmente importantes para determinar la calidad de la atención desarrollada por los voluntarios en el ámbito oncológico. Obtener información de los pacientes y sus familiares sobre aspectos muy específicos y puntuales de los diferentes servicios de voluntariado nos ayudará a rediseñar los procesos de atención para incrementar el nivel de satisfacción en el futuro. Es, por tanto, un enfoque metodológico que aporta gran riqueza de matices y que posibilita identificar "buenas prácticas" con las que incrementar la satisfacción de los usuarios, al tiempo que se identifican qué aspectos son los que más valoran y llaman su atención.

Este tipo de análisis permite identificar áreas de mejora en las cuales concentrar las acciones para conseguir mejoras en la prestación de los servicios de voluntariado. De esta forma se combina las valo- 
raciones de los usuarios sobre diferentes aspectos de los servicios recibidos con la importancia otorgada a cada uno de ellos. El trabajo de Calzada y colaboradores $^{(14)}$ realizado con voluntariado hospitalario también señala que este tipo de técnica de evaluación muestra una alta consistencia interna, unido a que es capaz de discriminar las diferentes áreas de mejora y que, es una técnica recomendable para que los gerentes sanitarios y sociales la utilicen cómo medida de calidad y eficacia en el entorno del trabajo de voluntariado oncológico.

En el presente estudio se realiza un análisis siguiendo las recomendaciones del modelo Análisis de Importancia-valoración (Importance-Performance Analisis) planteado originalmente por Martilla y James en $1977^{(21)}$. En primer lugar, los resultados de este trabajo permiten afirmar que existe un elevado nivel de satisfacción tanto en pacientes como en familiares con la atención recibida. Así, el 96\% de los usuarios se encuentran en los niveles superiores de satisfacción. Los aspectos que resultan más útiles a los beneficiarios son: apoyo emocional y acompañamiento durante la visitas a los pacientes ingresados. La valoración positiva también se manifiesta en la gran disposición a recomendar el contacto con los voluntarios. Así, un $77 \%$ se ha mostrado dispuesto a recomendar a otras personas el contacto con los voluntarios. Se ha obtenido un Índice NPS netamente positivo $(+80)$. Este dato resulta muy significativo dado que la literatura establece que un NPS superior a 0 se percibe como bueno y un NPS de 50 o más es excelente, así como una relación directa entre un alto NPS y el nivel de satisfacción de los usuarios. Los excelentes niveles de satisfacción y de recomendación, tienen una lectura inequívoca los voluntarios no defraudan las expectativas de pacientes y familiares.

El mapa de posicionamiento de las diferentes tareas atendiendo a la valoración que los usuarios realizan de las mismas y al porcentaje de importancia atribuido a cada una de ellas. Las tareas consideradas como especialmente importantes $y$ mejor valoradas son "Visitar a los enfermos para ofrecerles apoyo emocional" y "Suplir temporalmente a los familiares". Las actividades que tanto en importancia como en valoración han tendido a situarse alrededor del promedio general son aquellas cuyo objeto es ofrecer orientación e información. Concretamente, "Realización de gestiones y trámites hospitalarios", "Acompañar a los enfermos en los desplazamientos por el hospital" e "Informar sobre servicios AECC y otros recursos". Por último, las acciones que tanto en importancia como en valoración se sitúan por debajo de la media general son "Talleres educativos", "Carrito de acogida" y "Entretener a pacientes y familiares con actividades lúdicas". Es necesario realizar un análisis más detallado de estos datos y valorar las razones que hay detrás de dichas valoraciones. Destacar, que este resultado es contradictorio con los obtenidos en otros estudios realizados por la entidad donde el "desarrollo de talleres educativos" era la única actividad que correlaciona significativamente con el nivel de satisfacción $(p \geq 0,005)$ La explicación posible a estos datos contradictorios es que este tipo de actividades no se realiza en todos los hospitales, por lo tanto aquellos que no las conocen las valoran en menor medida.

Conocimiento y utilización de servicios de voluntariado son variables que inciden en la satisfacción de los pacientes y familiares. Es necesario desarrollar estrategias educativas y de promoción que nos permitan incrementar el nivel de conocimiento y de uso de las diferentes tareas por parte de los beneficiarios.

Por último, como áreas de mejora señalan una mayor difusión de la actividad, el incremento del número de voluntarios y la ampliación del horario de atención. 


\section{REFERENCIAS BIBLIOGRÁFICAS}

1. Fernández B, Yélamos C. Rehabilitación social del enfermo de cáncer. Rev Cáncer (Madrid) 2006;20:22-7.

2. Ministerio de Sanidad y Consumo. Estrategia en Cuidados Paliativos del Sistema Nacional de Salud 2007.p. 74.

3. Asociación Española Contra el Cáncer. Manual de Voluntarios. Cuidados paliativos de enfermos oncológicos terminales. Atención domiciliaria. Madrid, 1994.

4. Lacasta MA, Sastre P. Formación del voluntariado en cuidados paliativos. Med Paliat 2000;7:14-6.

5. Montesinos F. Nuevos retos en la formación del voluntariado en cuidados paliativos. Med Paliat 2008;15(Supl ):69-70.

6. Glanz K, Lerman C. Psychosocial impact of breast cancer: A critical review. Ann Behav Med 1992;14:204-12.

7. Moyer A, Salovey P. Psychosocial sequelae of breast cancer and its treatment. Ann Behav Med 1996;18:110-25.

8. Hann D, Baker F, Denniston M. The influence of social support on depressive symptoms in cancer patients: Age and gender differences. J Psychosom Res 2002;52: 27983. Doi:10.1016/S0022-3999(01)00235-5

9. Stark D, Kiely M, Smith A, Velikova G, House A, Selby P. Anxiety disorders in cancer patients: The nature, associations, and relation to quality of life. J Clin Oncol. 2002;20:3137-48. Doi: 10.1200/ JCO.2002.08.549

10. De Leeuw JRJ, De Graeff A, Ros WJG, Hordijk GJ, Blijham GH, Winnubst JAM. Negative and positive influences of social support on depression in patients with head and neck cancer: A prospective study. Psychooncology 2000;9:20-8. Doi: 10.1002/ (SICI)1099-1611(200001/02)9:1<20::AIDPON425>3.0.CO;2-Y

11. Koopman C, Hermanson K, Diamond S, Angell K, Spiegel D. Social support, life stress, pain and emotional adjustment to advanced breast cancer. Psychooncology
1998;7:101-11. Doi: 10.1002/(SICI)1099$1611(199803 / 04) 7: 2<101:$ :AID PON299>3.0.CO;2-3

12. Trunzo JJ, Pinto BM. Social support as a mediator of optimism and distress in breast cancer survivors. J Consult Clin Psychol 2003;71:805-11. Doi:10.1037/0022006X.71.4.805

13. Devine D, Parker PA, Fouladi RT, Cohen L. The association between social supportt, intrusive thoughts, avoidance and adjustment following an experimental cancer treatment. Psychooncology 2003;12:45362. Doi: 10.1002/pon.656

14. Gil, Carrasco A, Muñoz V. Evaluación de la calidad de un servicio de voluntariado en oncología: un Análisis Importancia-Valoración (IPA). Psicooncología 2016;13:71-83. Doi: 10.5209/rev_PSIC.2016.v13.n1.52488

15. Mira JJ, Lorenzo S, Rodríguez-Marín J, Aranaz J, Sitges E. La aplicación del modelo europeo de gestión de la calidad total del sector sanitario: ventajas y limitaciones. Rev Calidad Asistencial 1998;13;92-7

16. Mira, JJ, Rodríguez-Marín J, Peset R, Ybarra J, Perez-Jover V, Palazon I, et al. Causas de satisfacción y de insatisfacción en los pacientes en hospitales y atención primaria. Rev Calidad Asistencial 2002;17; 273-83

17. Mira JJ, Aranaz J, Lorenzo S, RodríguezMarín J, Moyano S. Evolución de la calidad percibida por los pacientes de dos hospitales públicos. Psicothema 2001;13:581-5.

18. Murillo C, Saurina C. Medida de la importancia de las dimensiones de la satisfacción en la provisión de servicios de salud. Gac Sanit 2013;27:304-9. Doi: 10.1016/j. gaceta.2012.12.015

19. Varela J, Rial A, García E. Presentación de una Escala de Satisfacción con los Servicios Sanitarios de Atención Primaria. Psicothema 2003;15;656-61

20. Reichheld FF. The one number you need to grow. Harv Bus Rev 2003;84:46-54

21. Martilla JA, James JC. Importance-performance analysis. J Marketing 1977;41:77-

9. Doi: $10.2307 / 1250495$ 